\title{
Vulnerabilidad climática de los sistemas de producción cafetalera en la zona de Cocoyagua en el departamento de Copán, Honduras, 2014-2015
}

Juan Manuel López Guevara

\section{RESUMEN}

La investigación analiza la vulnerabilidad ante amenazas y perturbaciones climáticas de los sistemas de producción cafetalera dentro del área protegida de Cocoyagua, en el departamento de Copán, Honduras, en la temporada 2014-2015, en función de la determinación de su sensibilidad, exposición y capacidad de adaptación, identificando estrategias de adaptación y su viabilidad de aplicación en los sistemas de producción.

La estrategia de diseño de la investigación es no experimental, aplicándose técnicas e instrumentos de medición para la obtención de la información. Las unidades de análisis del estudio están constituidas por las familias de productores de café y sus fincas cafetaleras. Debido al tamaño relativamente pequeño de la población a estudiar, no se calculó la muestra, ya que esta resultaba muy cercana al total de la población. La investigación se enmarca en la conceptualización del Grupo Intergubernamental de Expertos sobre el Cambio Climático (IPCC, 2007), en la cual la vulnerabilidad es una función del carácter, magnitud y proporción del cambio climático y la variación a la cual un sistema está expuesto, su sensibilidad y su capacidad de adaptación.

Se encontraron altos índices de exposición y sensibilidad y una baja capacidad de adaptación ante las amenazas y perturbaciones climáticas. Los recursos sociales muestran las condiciones más precarias de adaptación, lo que a su vez incide en los demás recursos naturales, físicos, financieros y humanos de la zona. En general, las estimaciones indican una alta vulnerabilidad de los sistemas de producción cafetalera de la zona ante los efectos del cambio climático.

${ }^{1}$ Profesor beneficiario de una beca básica, Escuela de Ingenieria, Centro Universitario Regional de Occidente (CUROC), UNAH: lopezgjm@gmail.com. 
Palabras claves: vulnerabilidad, sistemas de producción de café, cambio climático, amenazas climáticas, exposición, sensibilidad, capacidad de adaptación.

\section{ABSTRACT}

The research assesses the vulnerability to climate hazards and perturbations of the coffee production systems in a protected area named Cocoyagua, at the department of Copan, Honduras, during the crop season of 2014-2015, depending on the determination of its sensitivity, exposure and adaptive capacity, identifying adaptation strategies and the feasibility of application in the production systems. The research design strategy is non-experimental, using surveying techniques and instruments for the collection of data. The units of analysis are constituted by the coffee farmers' families and their coffee farms. Due to the relatively small size of the study population, the sample number resulted close to the total population, therefore, was not calculated.

The research adopted a conceptual framework based on the IPCC assessments (2007), in which vulnerability "is a function of the character, magnitude and rate of climate change and variation to which a system is exposed, its sensitivity and its low adaptive capacity". High exposure and sensitivity, and low adaptive capacity to climate hazards and perturbations indexes were found. Social resources show the most precarious adaptation conditions, adding to their situation, their influence over the other natural, physical, financial and human resources in the zone. In general, estimations indicate a high vulnerability of the coffee production systems in the zone to climate change effects.

Key words: vulnerability, coffee crop systems, climate change, climate hazards, exposure, sensitivity, adaptive capacity. 


\section{INTRODUCCIÓN}

El cultivo del café es el principal rubro agrícola generador de ingresos para las familias de la región occidental de Honduras, en el cual intervienen muchos productores individuales, empresas familiares, cooperativas, comercializadoras y exportadoras. En los últimos años el rubro cafetalero ha sufrido varias crisis, las cuales han causado estragos en las fincas y, consecuentemente, en las familias productoras. Devastadores han sido los efectos de la enfermedad causada por el hongo de la roya (Hemileia vastatrix), que según estudios de expertos, como el Instituto Interamericano de Cooperación para la Agricultura, Programa Cooperativo Regional para el Desarrollo Tecnológico y la Modernización de la Caficultura (IICA y PROMECAFE, 2013) y la Comisión Económica para América Latina y el Caribe y Consejo Agropecuario Centroamericano del Sistema de la Integración Centroamericano (CEPAL y CAC/SICA, 2014) está relacionada con la variabilidad climática y el aumento de la temperatura media en los últimos años, factores que habrían generado condiciones favorables para el brote del patógeno. La problemática que se presenta en los sistemas de producción está asociada al manejo de los recursos naturales y la degradación de los ecosistemas.

Los estudios de análisis de la vulnerabilidad ante la variabilidad y el cambio climático, particularmente sobre los sistemas de producción cafetalera, son escasos o inexistentes en la región occidental del país. Se distinguen importantes vacíos de conocimiento relacionados con el análisis de los impactos climáticos, sus tendencias y la modelación de escenarios futuros del comportamiento de los cultivos, la comprensión de los medios de vida y de los recursos de las comunidades, de las organizaciones y políticas relacionadas con los sistemas de producción. La poca disponibilidad de información climática e hidrométrica y, concretamente, la inexistencia de registros cronológicos de datos, así como de datos de parámetros de suelos, cobertura vegetal, rendimientos de los cultivos e información sobre el uso y gestión del agua, limitan la implementación de modelos de análisis.

El estudio se enfoca en analizar la vulnerabilidad de los sistemas de producción de café que se encuentran dentro de la zona de protección forestal Cocoyagua, que en los últimos años han sufrido los efectos de los cambios en los regímenes de precipitaciones, la incidencia de plagas y enfermedades, con las subsecuentes manifestaciones en la economía de las familias productoras. Así, se pretende proponer elementos que sirvan para la protección y conservación de los sistemas de producción de la zona ante los impactos de las amenazas climáticas, fortaleciendo la capacidad de 
adaptación de los recursos en términos de su acceso, flexibilidad y estabilidad.

Se espera que los resultados sirvan como elementos para la toma de decisiones en el diseño y manejo de sistemas de producción cafetalera sostenibles y amigables con el ambiente; en la prevención, control y mitigación de desastres relacionados con las amenazas identificadas, especialmente en beneficio de las familias productoras de café de la zona, pudiendo ser utilizados por investigadores, instituciones de desarrollo, municipalidades u otra entidad vinculada con el manejo de los sistema de producción.

Para tales fines, se construyó una herramienta de apoyo para evaluar los impactos de la variabilidad y el cambio climático a nivel de los sistemas de producción, cuyo marco conceptual se sustentó en los reportes de evaluación climática del IPCC (2007) y en el enfoque de medios de vida sostenibles para el análisis de la capacidad de adaptación (DFID, 2001). Estudios de análisis de vulnerabilidad realizados por instituciones como el Centro Agronómico Tropical de Investigación y Enseñanza (CATIE) y el Centro Internacional de Agricultura Tropical (CIAT) en otros lugares de Latinoamérica (Baca, 2011; Baca y otros, 2011y 2012; Programa Conjunto de Integración de Ecosistemas y Adaptación al Cambio Climático en el Macizo Colombiano, 2011; Altamirano, 2012; Baca, Läderach, Haggar, Schroth y Ovalle, 2014), han brindado aportes metodológicos importantes para la construcción de los instrumentos y determinación de los indicadores en los factores analizados.

La situación de los sistemas de producción de café de la zona de protección forestal Cocoyagua, con relación a otras zonas productoras de la región occidental del país, además de su condición como área de vocación forestal, se consideraron como criterios para enfocar la investigación en la zona mencionada.

\section{METODOLOGÍA}

\section{Diseño}

La investigación presenta un carácter cuantitativo que parte de un problema de estudio delimitado y concreto, en el cual la recolección de los datos se fundamenta en la medición de variables en el contexto de la zona de Cocoyagua, utilizando métodos y procedimientos estandarizados para la obtención de datos y para el análisis estadístico. Se desarrolla un proceso lógico y estructurado para el diseño, 
recolección y análisis de la información de la población de familias productoras y sus sistemas de producción de la zona de estudio. La investigación es no experimental, ya que es ajena a cualquier control o manipulación intencional y solamente se analiza una situación existente previamente identificada y delimitada, aplicando instrumentos para recolectar información en el núcleo de las familias y sus fincas de café.

Se considera como un estudio transversal, ya que se recolectaron datos en un solo período de tiempo durante el año 2015, para describir variables relacionadas con las condiciones que determinan la vulnerabilidad de los sistemas de producción de café ante los efectos de amenazas y perturbaciones climáticas de los sistemas de producción en la zona mencionada.

\section{Población sobre la que se ha hecho el estudio}

El universo del estudio está constituido por unos 108 productores y sus unidades de producción cafetalera ubicados en el área de la zona protegida Cocoyagua, ubicada en la parte central del departamento de Copán, en el occidente de Honduras. Las fincas abarcan unas 356 hectáreas de café dentro de la zona.

Fue necesario hacer ajustes en el tamaño de la población de productores a estudiar, ya que no existía un inventario actualizado en las juntas locales de productores de café, ni en ninguna otra fuente. Los datos surgieron de la información proporcionada en visitas previas a la presentación de la propuesta. No obstante, al proceder a recolectar la información, se pudo constatar que muchos productores no habían sido identificados preliminarmente o que otros que se presumía habían abandonado sus fincas a raíz de la epidemia de la roya en el año 2012, aún subsistían dentro del sistema de producción. Las unidades de análisis del estudio están representadas por cada una de las familias y sus fincas cafetaleras que se encuentran en el área referida a las que se aplicaron los instrumentos de medición. Se determinó estudiar toda la población, ya que no se consideró necesario calcular la muestra, dado que la población a estudiar es pequeña y al efectuar el cálculo de la muestra esta resultaba muy cercana al total de la población.

\section{Entorno}

La zona Cocoyagua se encuentra localizada en el occidente de Honduras, dentro del área geográfica de los municipios de Veracruz, San José, Dolores y Trinidad, del departamento de Copán. En el área se encuentran las comunidades El Ocote, El Triunfo, Platanitos, Mecederos, San Antonio, Yaruconte, Pasquingual, Eramola y 
Quebraditas, en alturas que van desde los 900 hasta los 1490 msnm.

La zona posee acuerdo de declaratoria de área de vocación forestal y se encuentra en proceso de demarcación. Comprende 878.95 hectáreas y está constituida por diferentes tipos de vegetación, predominando el bosque latifoliado, el cultivo del cafeto, los pastos y granos básicos. Sus fuentes de agua abastecen la mayor parte de la demanda de la población de los municipios mencionados.

\section{Intervenciones}

La técnica a través de la cual se estableció la relación con las unidades de análisis fue la encuesta personal. Por medio de esta los encuestadores, previamente capacitados, aplicaron los instrumentos de medición (cuestionarios) que se diseñaron de manera estructurada, estandarizada, planteando preguntas cerradas con igual número de opciones con un mismo peso para todas, entre las que se debía seleccionar una única respuesta.

Las puntuaciones de la escala se obtuvieron sumando los valores alcanzados respecto de cada pregunta. Según Hernández, Fernández y Collado (2010), se denomina escala aditiva, pudiéndose calificar, además, el promedio resultante con la suma de las puntuaciones en la escala y el número de preguntas. La medición es ordinal, en una escala que permite clasificar las respuestas en puntuaciones ordenadas, en rangos o escalas cuyas respuestas presentan dirección, desde una perspectiva favorable, pasando por los valores medios hasta el extremo desfavorable. Como lo plantea Ritchey (2008, p. 44), las respuestas van "desde la mayor hasta la menor, de la mejor a la peor, de la primera a última". De esa manera, se obtuvo información cuantitativa que posteriormente pudiera ser tabulada y analizada estadísticamente.

\section{ANÁLISIS DE LOS DATOS}

Por medio de los procedimientos descritos anteriormente, se construyeron escalas para cada productor, comunidad y la zona de estudio. Luego, se determinó la vulnerabilidad (V) de los sistemas de producción, calculándola en función de la exposición (E), la sensibilidad (S) y la capacidad de adaptación (V = f [E, S, A]), (Brooks, 2003; IPCC, 2007; Johnston y Williamson, 2007; Vincent, 2010; Lampis, 2013). Se determinaron valores de 0-1 para una vulnerabilidad baja, de 1-2 vulnerabilidad media y 2-3, vulnerabilidad alta. 
La tabulación y el procesamiento de los datos obtenidos se realizaron utilizando hojas de cálculo de Microsoft Excel, aplicando funciones estadísticas descriptivas y reglas de formato condicional, obteniendo tablas y gráficos para la presentación de la información.

Los métodos numéricos que se utilizaron para describir los datos cuantitativos fueron las medidas de tendencia central, para determinar los índices de cada una de las dimensiones de las variables y para obtener los índices de exposición, sensibilidad y capacidad de adaptación para cada uno de los productores, comunidades y para la zona en general. Los métodos gráficos empleados fueron principalmente los gráficos de sectores y los radiales. Los primeros muestran la contribución de cada factor a un total (la distribución porcentual), mientras que los otros señalan valores relativos al punto central (distribución de los índices para cada uno de los recursos).

Los índices se clasificaron en rangos o escalas, construidos a partir de reglas de formato condicional en EXCEL, simbolizados con colores verde, amarillo y rojo, para determinar una perspectiva favorable, media o desfavorable.

Adicionalmente se realizó un análisis de varianza (ANOVA) para determinar si la variabilidad que existe en los datos de las estimaciones de los índices de vulnerabilidad entre las comunidades se deben al azar 0 a las diferencias entre las poblaciones de las que proceden las muestras.

\section{RESULTADOS}

Exposición de los sistemas de producción cafetalera en la zona de protección forestal Cocoyagua

La percepción general es que el clima ha cambiado en la zona en los últimos años, siendo los cambios en la temperatura y en el régimen de las lluvias las perturbaciones más importantes. El índice obtenido (ver cuadro 1), indica una alta exposición de la zona a los cambios y variaciones climáticas, manifestada en pérdidas de plantas, incidencia de plagas y enfermedades y alteración en los ciclos fenológicos de las plantas. 


\section{Cuadro 1. Carácter de los cambios y variaciones climáticas}

\begin{tabular}{|l|l|c|}
\hline \multicolumn{1}{|c|}{ Indicador } & \multicolumn{2}{c|}{ Índice de exposición } \\
\hline Exposición a las heladas & 2.2685 & \\
\hline Cambios en el régimen de lluvias & 2.5185 & \\
\hline Exposición a las sequías & 1.5926 & \\
\hline Exposición a los vientos & 1.7500 & \\
\hline Cambios en la temperatura & 2.7593 & \\
\hline Promedio & 2.2870 & \\
\hline
\end{tabular}

Fuente: elaboración propia.

Los efectos a nivel de finca muestran una mayor manifestación en las enfermedades, probablemente relacionadas con los cambios en las temperaturas y las alteraciones en los regímenes de las precipitaciones, aunque también exacerbados por las prácticas inadecuadas de manejo de los suelos y los cultivos. En general, también se presenta una alta exposición ante los efectos relacionados con el clima (ver cuadro 2).

\section{Cuadro 2. Efectos relacionados con el clima en las fincas}

\begin{tabular}{|l|l|r|}
\hline \multicolumn{1}{|c|}{ Indicador } & \multicolumn{2}{c|}{ Índice de exposición } \\
\hline Exposición a los deslizamientos & 1.9259 & \\
\hline Exposición a plagas & 2.0926 & \\
\hline Exposición a enfermedades & 2.5278 & \\
\hline Defoliación de los cafetos & 2.2500 & \\
\hline Cambios en la floración de las plantas & 2.1667 & \\
\hline Caída de flores y frutos & 2.0000 & \\
\hline Promedio & 2.1605 & \\
\hline
\end{tabular}

Fuente: elaboración propia.

La característica menos favorable del suelo de las fincas es la pendiente, que en un $95 \%$ es muy inclinada, favoreciendo la pérdida de suelos, erosión y nutrientes con el agua. Otras, como la fertilidad, pedregosidad, profundidad y capacidad de infiltración, presentan índices de sensibilidad regular (ver cuadro 3). 


\section{Cuadro 3. Características del suelo de las fincas y su sensibilidad}

\begin{tabular}{|l|l|c|}
\hline \multicolumn{2}{|c|}{ Indicador } & \multicolumn{2}{c|}{ Índice de sensibilidad } \\
\hline Pendiente del terreno & 2.9537 & $\mathbf{D}$ \\
\hline Fertilidad del suelo & 1.5185 & $\mathbf{O}$ \\
\hline Pedregosidad superficial & 1.1759 & $\mathbf{O}$ \\
\hline Grado de erosión & 1.8981 & $\mathbf{O}$ \\
\hline Profundidad del suelo & 1.9352 & $\mathbf{O}$ \\
\hline Capacidad de infiltración & 1.9444 & $\mathbf{O}$ \\
\hline Promedio & 1.9043 & $\mathbf{O}$ \\
\hline
\end{tabular}

Fuente: elaboración propia.

La ausencia de prácticas de conservación de suelos en las fincas representa altos niveles de sensibilidad (ver cuadro 4), ya que aproximadamente el $82 \%$ de los productores no implementa ninguna práctica de conservación.

\section{Cuadro 4. Prácticas de manejo del suelo en las fincas y su sensibilidad}

\begin{tabular}{|l|l|r|}
\hline \multicolumn{1}{|c|}{ Prácticas de manejo } & \multicolumn{2}{c|}{ Índice de sensibilidad } \\
\hline Frecuencia de fertilización química & 2.1759 & \\
\hline Frecuencia de fertilización orgánica & 2.8426 & \\
\hline Manejo de cobertura de mulch & 1.9259 & \\
\hline Prácticas de conservación de suelos & 2.8056 & \\
\hline Promedio & 2.4375 & \\
\hline
\end{tabular}

Fuente: elaboración propia.

Las prácticas de manejo del cultivo que muestran los niveles más sensibles son el manejo de residuos de la cosecha (2.6296), los controles fitosanitarios (2.4), el manejo de la sombra (2.0926) y de tejidos (2.3148), lo que refleja bajos niveles en conocimientos y adopción de técnicas de manejo y poca motivación para realizar prácticas innovadoras en las fincas. Apenas el $4 \%$ de los productores maneja adecuadamente los residuos, no hay estrategias integradas y sostenibles de manejo fitosanitario y las prácticas de manejo de la sombra y de tejidos en el cultivo no son efectuadas adecuadamente por la mayoría de los productores (ver figura 1). 
Ciertamente, la alta sensibilidad (2.1528) está relacionada con las prácticas inadecuadas que los agricultores realizan en sus suelos y con el manejo del cultivo, estos a su vez contribuyen a aumentar la degradación y contaminación de los sistemas con el peligro para el ambiente y la salud humana.

Figura 1. Distribución de las prácticas de manejo del cultivo en las fincas

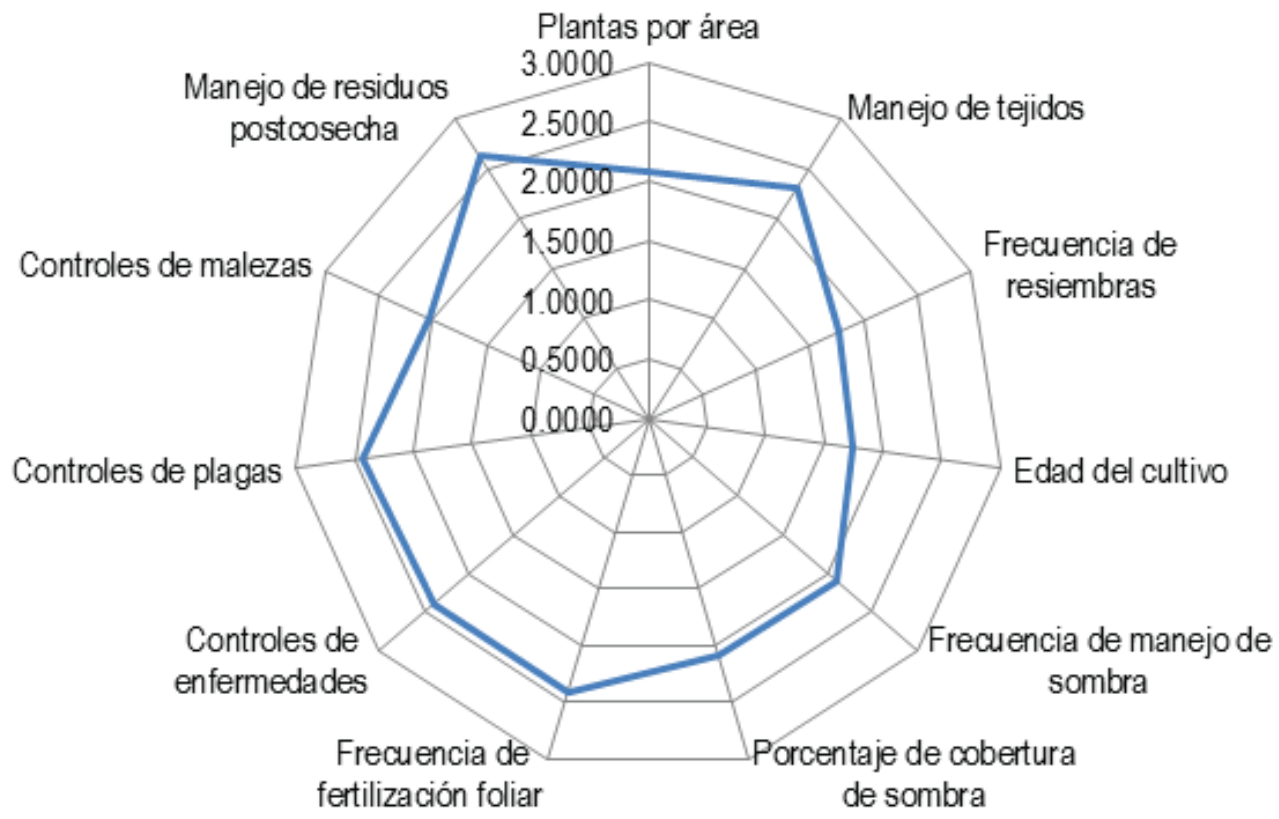

Fuente: elaboración propia.

Capacidad de adaptación de los sistemas de producción de café en la zona de Cocoyagua

Entre los recursos naturales analizados, el recurso agua muestra un índice de regular capacidad de adaptación, mientras la tierra y el bosque muestran una baja capacidad de adaptación. Los índices más bajos dentro del recurso agua se encuentran relacionados con la poca diversidad de fuentes en la zona y con el acceso y utilización limitados del agua para actividades productivas. Con respecto al recurso tierra, los índices más bajos se presentan con relación al uso de tecnologías de apoyo a la producción, ya que son totalmente inexistentes (2\%). Los índices más bajos relacionados al 
recurso bosque se evidencian en la inaccesibilidad a los productos forestales y la inexistencia de planes para regular los servicios del bosque.

Se distinguen condiciones críticas que determinan la situación del acceso, flexibilidad y estabilidad de los recursos naturales (ver cuadro 5), como la deficiente capacidad excedentaria relacionada con la pérdida de la capacidad productiva, la inequidad en la distribución, los bajos niveles tecnológicos y la poca diversificación.

Cuadro 5. Índices de capacidad de adaptación de los recursos naturales

\begin{tabular}{|l|c|c|c|c|c|}
\hline Recurso & Índice de acceso & $\begin{array}{l}\text { Índice de } \\
\text { flexibilidad }\end{array}$ & $\begin{array}{l}\text { Índice de } \\
\text { estabilidad }\end{array}$ & \multicolumn{2}{|l|}{ Índice de capacidad de adaptación } \\
\hline Agua & 1.6273 & 1.9938 & 1.5972 & 1.7395 & \\
\hline Tierra & 1.9568 & 2.6057 & 1.9352 & 2.1659 & \\
\hline Bosque & 2.2130 & 1.9907 & 2.0741 & 2.0926 & \\
\hline Promedio & 1.9324 & $\mathbf{2 . 1 9 6 8}$ & 1.8688 & 1.9993 & \\
\hline
\end{tabular}

Fuente: elaboración propia.

También se muestran condiciones escasas de la infraestructura para prestación de servicios a los caficultores, como la ausencia de facilidades y alternativas para el beneficiado, indicando una baja capacidad de adaptación de los recursos físicos (ver cuadro 6). La ausencia de tecnologías alternativas para el secado y para otros procesos de la poscosecha, muestran debilidades de adaptación con respecto al aprovechamiento de energías renovables, el manejo de desechos y la reducción de la contaminación.

Cuadro 6. Índices de capacidad de adaptación de los recursos físicos

\begin{tabular}{|l|l|l|l|l|c|}
\hline Recurso & $\begin{array}{l}\text { Índice de } \\
\text { acceso }\end{array}$ & $\begin{array}{l}\text { Índice de } \\
\text { flexibilidad }\end{array}$ & $\begin{array}{l}\text { Índice de } \\
\text { estabilidad }\end{array}$ & \multicolumn{2}{|l|}{ Índice de capacidad de adaptación } \\
\hline Caminos & 1.8519 & 2.0000 & 2.0370 & 1.9630 & \\
\hline Medios de transporte & 2.1019 & 2.1944 & 2.0556 & 2.1173 & \\
\hline Servicios poscosecha & 2.2361 & 2.6102 & 3.0000 & 2.6154 & \\
\hline Promedio & $\mathbf{2 . 0 6 3 3}$ & $\mathbf{2 . 2 6 8 2}$ & $\mathbf{2 . 3 6 4 2}$ & $\mathbf{2 . 2 3 1 9}$ & \\
\hline
\end{tabular}

Fuente: elaboración propia. 
La baja capacidad de adaptación de los recursos financieros (ver cuadro 7) está vinculada con la ausencia de mecanismos para propiciar un acceso justo a los mercados, a fuentes de ingreso y al crédito. El acceso a los mercados presenta los más bajos índices de adaptación, ya que la única opción que tienen son los intermediarios a quienes venden sus productos bajo condiciones totalmente desfavorables. La difícil situación de inestabilidad en los ingresos está muy asociada con los mercados, en la cual los productores se encuentran a merced de las fluctuaciones de los precios del café, cuyos impactos son acentuados porque la mayoría depende, además del café, de cultivos de subsistencia que también son afectados por las perturbaciones climáticas.

\section{Cuadro 7. Índice de capacidad de adaptación de los recursos financieros}

\begin{tabular}{|l|c|c|c|c|c|}
\hline Recurso & $\begin{array}{l}\text { Índice de } \\
\text { acceso }\end{array}$ & $\begin{array}{l}\text { Indice de } \\
\text { flexibilidad }\end{array}$ & $\begin{array}{l}\text { Índice de } \\
\text { estabilidad }\end{array}$ & \multicolumn{3}{|c|}{ Índice de capacidad de adaptación } \\
\hline Ingresos & 2.2151 & 2.0327 & 2.7377 & 2.3285 & $\mathbf{D}$ \\
\hline Crédito & 2.1574 & 2.3102 & 2.2685 & 2.2454 & $\mathbf{D}$ \\
\hline Insumos externos & 1.7870 & 1.8056 & 1.8796 & 1.8241 & $\mathbf{D}$ \\
\hline Mercados & 2.9167 & 2.0556 & 2.6574 & 2.5432 & $\mathbf{D}$ \\
\hline Promedio & $\mathbf{2 . 2 6 9 0}$ & $\mathbf{2 . 0 5 1 0}$ & $\mathbf{2 . 3 8 5 8}$ & $\mathbf{2 . 2 3 5 3}$ & $\mathbf{D}$ \\
\hline
\end{tabular}

Fuente: elaboración propia.

Los bajos índices de capacidad de adaptación que presentan los recursos sociales, muestran la carencia de organizaciones y procesos comunitarios y la ausencia institucional en la zona, lo que reduce las posibilidades de los productores de acceder a mercados competitivos, a financiamiento y a otros beneficios propiciados por la colectividad. Las condiciones sociales en que se encuentran las familias productoras contribuyen a acentuar la vulnerabilidad en otros recursos de los sistemas de producción (ver cuadro 8).

Los recursos humanos también manifiestan su baja capacidad de adaptación por la ausencia de oportunidades para capacitaciones, asistencia técnica u otras opciones de fortalecimiento de sus capacidades. Los recursos políticos muestran una baja capacidad de adaptación, reflejada en la poca capacidad de gestión, de acceso a la toma de decisiones y a las relaciones de poder. 
Cuadro 8. Índices de capacidad de adaptación de los recursos sociales, humanos y otros recursos

\begin{tabular}{|l|c|c|c|c|c|}
\hline Dimensión & $\begin{array}{l}\text { Índice de } \\
\text { acceso }\end{array}$ & $\begin{array}{l}\text { Índice de } \\
\text { flexibilidad }\end{array}$ & $\begin{array}{l}\text { Índice de } \\
\text { estabilidad }\end{array}$ & \multicolumn{2}{|c|}{ Índice de capacidad de adaptación } \\
\hline Recursos sociales & 2.2407 & 2.7593 & 2.8333 & 2.6111 & \\
\hline Recursos humanos & 2.0556 & 2.5185 & 1.7917 & 2.1219 & \\
\hline $\begin{array}{l}\text { Otros recursos } \\
\text { (culturales, políticos) }\end{array}$ & 2.4815 & 2.9630 & & 2.7222 & \\
\hline
\end{tabular}

En general, se refleja una baja capacidad de adaptación en todos los recursos de apoyo para la producción cafetalera en la zona, entre los cuales, los recursos sociales y los políticos muestran los índices más críticos. La distribución de los valores relativos de los índices de acceso, flexibilidad y estabilidad de los recursos se muestra en la figura 2.

Figura 2. Distribución de los índices de acceso, flexibilidad y estabilidad de los recursos analizados

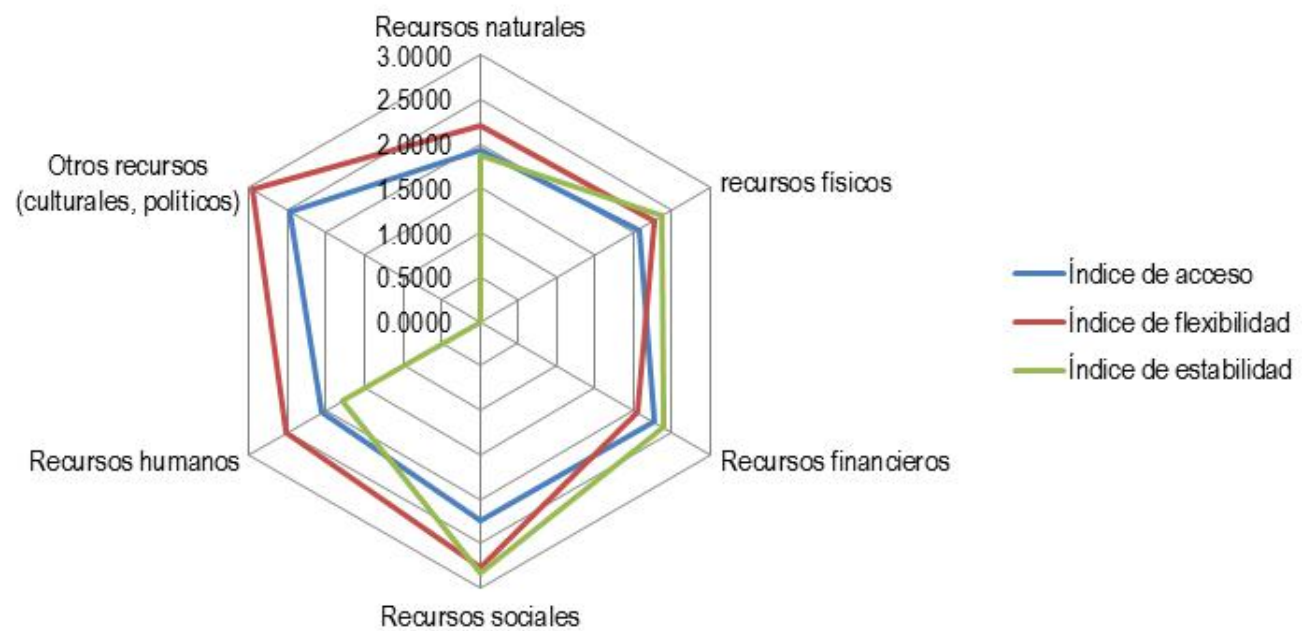

Fuente: elaboración propia. 


\section{Vulnerabilidad de los sistemas de producción}

Se obtuvieron estimaciones altas de vulnerabilidad (2.1880) de los sistemas de producción cafetalera de la zona, manifestadas, a su vez, por una alta exposición (2.2238), una alta sensibilidad (2.1528) y una baja capacidad de adaptación (2.1886) ante las amenazas y perturbaciones climáticas.

Además, la comparación de las condiciones de alta vulnerabilidad que presenta cada una de las comunidades muestra que la problemática que enfrentan es bastante homogénea, en cuanto a los factores de exposición, las debilidades intrínsecas de sus sistemas y su escasa capacidad de enfrentar las amenazas, ya que no existe variación estadística entre las estimaciones medias de vulnerabilidad obtenidas de cada una de las comunidades (ver cuadro 9).

\section{Cuadro 9. Tabla de ANOVA}

\begin{tabular}{|l|c|l|l|l|c|}
\hline Fuente de variación & $\begin{array}{l}\text { Grados de } \\
\text { libertad }\end{array}$ & $\begin{array}{l}\text { Suma de } \\
\text { cuadrados }\end{array}$ & $\begin{array}{l}\text { Cuadrado } \\
\text { medio }\end{array}$ & F calculado & F tabulado \\
\hline Entre tratamientos & 6 & 0.33087968 & 0.05514661 & \multirow{2}{*}{0.5857521} & 2.17 \\
${$\cline { 1 - 5 }$} }$ & 102 & 9.60296101 & 0.09414668 & 2.96 \\
\hline Total & 108 & 9.93384069 & - & - & - \\
\hline
\end{tabular}

Fuente: elaboración propia.

\section{DISCUSIÓN}

El estudio utiliza una herramienta para medir la vulnerabilidad, en la que se incluyen indicadores adaptados al contexto de los sistemas de producción de la zona que se utilizaron para realizar la cuantificación de los datos de cada uno de los ítems investigados y para determinar las estimaciones de los niveles de vulnerabilidad. Sin embargo, la descripción de los índices va más allá de los meros datos obtenidos, requiere un entendimiento del contexto local y de las vinculaciones con un entorno más amplio, a nivel regional y en otros ámbitos. 
Se proponen estrategias de adaptación al cambio climático enfocadas en el manejo de los diferentes recursos con que cuentan los sistemas de producción de la zona. Así, las intervenciones deberán garantizar la conservación de los recursos naturales y la biodiversidad, desarrollar estándares para validar buenas prácticas ambientales en la producción de café y garantizar la seguridad alimentaria de las familias cafetaleras y de la población. La atención a los recursos y servicios para la poscosecha debería ser prioritaria en el manejo de los recursos físicos, mientras que las estrategias con respecto a los recursos financieros deberían orientarse a priorizar opciones para propiciar un acceso justo y equitativo a los mercados y para incrementar la competitividad de los productores; pero, brindando opciones para la diversificación de las fincas familiares.

El fortalecimiento del capital humano y social, a través de la promoción de procesos y estructuras organizativas que favorezcan el desarrollo de las capacidades de los demás recursos de apoyo a los sistemas de producción, reviste capital importancia en la zona. El desarrollo de la capacidad de gestión, el acceso a la toma de decisiones y las relaciones de poder en las comunidades y municipios es imprescindible para mejorar las condiciones de los otros recursos analizados anteriormente. Las estrategias deben ser enfocadas a largo plazo y fundamentadas en bases científicas, estableciendo alianzas estratégicas con organismos de investigación y desarrollo.

La trascendencia de los resultados de la investigación es evidente para las familias cafetaleras de la zona de Cocoyagua, cuyos índices revelan condiciones precarias de alta vulnerabilidad que requieren el desarrollo de estrategias de adaptación enfocadas al manejo sostenible de los recursos naturales, físicos y financieros; pero con un énfasis en el desarrollo de las capacidades sociales, humanas y políticas para el manejo de los sistemas de producción.

No obstante, las repercusiones derivadas de los planteamientos metodológicos de la investigación pueden extenderse a otros contextos o regiones, en las que se pueden desarrollar acciones de replicación con las debidas consideraciones de ajuste y de mejoras de los instrumentos de recolección de datos.

El estudio se sustenta mayormente en la percepción de los productores de café con respecto a su situación actual, complementada con observaciones y mediciones de campo sobre indicadores relacionados con efectos derivados del clima, con prácticas de manejo y características agroecológicas de la zona y fincas. No pretende establecer tendencias o pronósticos de escenarios futuros de la situación de los sistemas cafetaleros de la zona, aunque sí puede proporcionar elementos de base para futuras 
evaluaciones de la vulnerabilidad.

Con el estudio se han identificado otras situaciones que requieren ser investigadas, como la determinación de la erosión en las laderas y su relación con las pérdidas de nutrientes y la fertilidad de los suelos. Estudios de la captura de carbono en los sistemas, la viabilidad de los sistemas agroforestales en la zona, el aprovechamiento de subproductos, entre otros, podrían desarrollarse para analizar los sistemas a mayor profundidad.

\section{CONCLUSIONES}

La percepción general es que el clima se ha alterado en la zona en los últimos años, manifestándose mayormente en cambios en la temperatura y en el régimen de las lluvias, cuyos efectos a nivel de finca se muestran en una mayor incidencia de enfermedades.

El índice de exposición revela altos impactos de las alteraciones climáticas, que a su vez son exacerbados por las condiciones de manejo de los sistemas de producción. La característica menos favorable de los terrenos de las fincas es la pendiente, cuya inclinación las hace susceptibles a la pérdida por erosión y de nutrientes con el agua. La ausencia de prácticas de conservación de suelos presenta altos niveles de sensibilidad. Los niveles más sensibles en el manejo del cultivo se encuentran en el tratamiento de los residuos de la cosecha, los controles fitosanitarios, el manejo de la sombra y de tejidos.

En cuanto al acceso, flexibilidad y estabilidad de los recursos naturales, los aspectos que inciden en su baja capacidad de adaptación son el deterioro de los recursos en los últimos años, manifestándose en una reducción de su capacidad y en la poca diversidad de prestación de sus servicios.

Las condiciones de los recursos físicos para la prestación de servicios a los caficultores son escasas, destacándose la ausencia de facilidades y alternativas para el beneficiado del café, las difíciles condiciones de los caminos y la falta de medios adecuados de transporte, lo que indica una baja capacidad de adaptación.

La baja capacidad de adaptación de los recursos financieros está en función de la escasez de opciones para acceder a los mercados, a fuentes de ingreso y al crédito. 
El índice más bajo de capacidad de adaptación en estos recursos se relaciona con el acceso y estabilidad de los mercados, ya que la única opción que tienen son los intermediarios a quienes venden sus productos bajo condiciones totalmente desfavorables.

La situación de los recursos sociales muestra la ausencia de organizaciones comunitarias y de instituciones de apoyo a los procesos de desarrollo de la zona. Por lo tanto, no hay oportunidades para el fortalecimiento de los recursos humanos. La poca capacidad de gestión, de acceso a la toma de decisiones y las relaciones de poder, se revela en los recursos políticos de las comunidades y los municipios de la zona. En general, se refleja una baja capacidad de adaptación en todos los recursos de apoyo para la producción cafetalera en la zona, entre los cuales los recursos sociales y los políticos muestran los índices más críticos, manifestándose en la inexistencia de procesos organizativos que favorezcan el desarrollo de las capacidades de los demás recursos de apoyo a los sistemas de producción que contribuyan a mejorar las capacidades productivas para movilizar recursos, para acceder a los mercados y a los servicios para la producción.

\section{AGRADECIMIENTOS}

Al Coordinador Regional de Investigación Científica del CUROC, Kevin Estévez, por todo el apoyo en la ejecución de la investigación. A la Dirección de Investigación Científica y Posgrados de la UNAH, por el apoyo para el desarrollo del estudio a través de la beca de investigación y la asesoría brindada.

\section{BIBLIOGRAFÍA}

Altamirano, J. (2012). Influencia de la variabilidad climática sobre la producción de café (Coffea arabica L.) en Honduras. Tesis. Costa Rica: CATIE.

Baca, M. (2011). Identificación de la vulnerabilidad en los medios de vida de las familias cafetaleras y sus posibles estrategias de adaptación al cambio climático en el norte de Nicaragua. Costa Rica: CATIE.

Baca, M.; Läderach, P.; Haggar, J.; Ovalle, O.; Ocón, S.; Gómez, L. y Zelaya, C. (2011). Vulnerabilidad y estrategias de adaptación al cambio climático en los medios de vida de las familias de Nicaragua. Nicaragua: Centro Internacional de 
Agricultura Tropical.

Baca, M.; Läderach, P.; Haggar, J.; Ovalle, O.; Ocón, S. y Gómez, L. (2012). Vulnerabilidad y estrategias de adaptación al cambio climático en los medios de vida de las familias de Nicaragua, El Salvador, Guatemala y México. Nicaragua: Centro Internacional de Agricultura Tropical.

Baca, M.; Läderach, P.; Haggar, J.; Schoth, G. y Ovalle, O. (2014). An integrated framework for assessing vulnerability to climate change and developing adaptation strategies for coffee growing families in Mesoamérica. PLoS ONE, 9(2), e88463.

Brooks, N. (2003). Vulnerability, risk and adaptation: a conceptual framework. Norwich: Tindall Centre for Climate Change Research.

Comisión Económica para América Latina y el Caribe [CEPAL] y Consejo Agropecuario Centroamericano del Sistema de la Integración Centroamericano y [CAC/SICA] (2014). Impactos potenciales del cambio climático sobre el café en Centroamérica. México: CEPAL.

Departamento para el Desarrollo Internacional. (2001). Guías sobre medios de vida sostenibles. Londres: DFID.

Grupo Intergubernamental de Expertos sobre el Cambio Climático. (2007). Cambio climático 2007: Informe de síntesis. Suiza: IPCC.

Hernández, R.; Fernández, C. y Baptista, M. (2010). Metodología de la Investigación. México: McGraw Hill Interamericana.

Instituto Interamericano de Cooperación para la Agricultura [IICA], Programa Cooperativo Regional para el Desarrollo Tecnológico y la Modernización de la Caficultura [PROMECAFE] (2013). La crisis del café en Mesoamérica, causas y respuesta apropiadas. Guatemala: IICA.

Johnston, M. y Williamson, T., (2007). A framework for assessing climate change vulnerability of the Canadian forest sector. The Forest Chronicle, 83(3).

Lampis, A. (2013). Vulnerabilidad y adaptación al cambio climático: debates acerca del concepto de vulnerabilidad y su medición. Revista Colombiana de Geografía, 22(2), 17-33.

Programa Conjunto de Integración de Ecosistemas y Adaptación al Cambio Climático en el Macizo Colombiano (2011). Análisis de vulnerabilidad y adaptación al cambio climático para la subcuenca río Molino (parte alta y media), municipio de Popayán. Colombia: Fondo para el Logro de los ODM.

Ritchey, F. (2008). Estadística para las ciencias sociales. México: McGraw-Hill Interamericana 